\title{
A Current Overview of Chronic Wounds Presenting to a Plastic Surgery Unit in Central India
}

This article was published in the following Dove Press journal: Chronic Wound Care Management and Research

\author{
Manal M Khan (iD \\ Ved Prakash Rao Cheruvu (D) \\ Deepak Krishna \\ Reena Minz \\ Michael Laitonjam \\ Rishabh Joshi $\mathbb{D}$
}

Department of Burns and Plastic Surgery, All India Institute of Medical Sciences, Bhopal, Madhya Pradesh, India
Correspondence: Ved Prakash Rao Cheruvu

Department of Burns and Plastic Surgery, All India Institute of Medical Sciences, Ist Floor, Hospital Building, Saket Nagar, Bhopal, Madhya Pradesh 462020, India Tel +9l 8872209777

Email vedprakash.

plasticsurg@aiimsbhopal.edu.in
Purpose: To analyze the demographic, clinical, and microbiological profile of patients presenting to our unit with chronic wounds of various etiologies with an intent to give a current overview of chronic wounds.

Patients and Methods: We performed a prospective observational study of patients presenting with chronic wounds from October 2018 to September 2019. The study was conducted at the Department of Burns and Plastic Surgery of a tertiary care institute in a nonmetropolitan city in Central India. A total of 103 patients were included in the study. Data collected from the patients included demographic details, history, clinical features, and relevant laboratory reports. Wound swabs obtained by Levine's technique were sent for culture and sensitivity studies. Treatment was instituted according to the clinical picture and modified if necessary. Progress was monitored until the wound healed, either by conservative management or by surgical intervention. Patients were followed up for six months thereafter. Results: Most of the patients presented with lower limb wounds ( $n=81,78.64 \%$ ). Swab specimens from 103 wounds were cultured. Among the isolates, gram-negative organisms were more common than gram-positive organisms. Staphylococcus aureus was the most common species isolated, followed by Pseudomonas aeruginosa. The frequency of infections caused by other gram-negative organisms like Klebsiella pneumoniae, Escherichia coli, and Proteus mirabilis was on the rise. There were significant differences in the patterns of antimicrobial resistance in our patients. Sharp debridements were required in almost all cases for wound preparation. Most of the patients $(n=74,71.84 \%)$ underwent surgical intervention for achieving wound closure. Split-thickness skin grafting (STSG) was the most common surgical intervention performed ( $n=45,43.68 \%$ patients), followed by local and distant flaps.

Conclusion: Our study gives a current overview of the causes, clinical presentation, prevalent microbial flora, and their antibiotic susceptibilities prevalent in chronic wounds presenting to our unit. Treatments administered are discussed with emphasis on the different reconstructions performed.

Keywords: anti-bacterial agents, biofilms, drug resistance, multiple bacterial, Pseudomonas aeruginosa, Staphylococcus aureus, wound healing

\section{Introduction}

Chronic wounds are often painful, debilitating and profoundly impair the quality of life of the affected individuals. ${ }^{1}$ They impose an enormous economic burden on the patients and healthcare systems around the world. A study estimated that there were 2.2 million patients in the United Kingdom with a wound, equating to $4.5 \%$ of the 
adult population. This led to 3.4 million hospital outpatient visits annually and a cost of $£ 5.3$ billion for managing these wounds and the associated comorbidities. ${ }^{2}$ According to some estimates, chronic wounds of the lower extremity affected 2.4-4.5 million people in the United States. ${ }^{3,4}$ Chronic wound infections caused approximately $85 \%$ of all non-traumatic lower-limb amputations and $7-8 \%$ of fatalities in spinal cord injury victims. ${ }^{1,5,6}$

Wound healing involves a cascade of complex and dynamic processes that can be affected by various factors such as elderly age, underlying diseases (venous incompetence, diabetes, and arterial insufficiency), obesity, medications, poor nutrition, and infection resulting in a chronic wound. ${ }^{7}$ Several factors affect the wound's ability to progress from the inflammatory phase to the proliferation phase. ${ }^{8}$ These include the underlying pathology, presence of non-viable tissue, and abnormal immune cell activity, which results in an excessive release of MMPs (matrix metalloproteinases). This process perpetuates the cycle of wound chronicity and extracellular matrix destruction. ${ }^{9}$ Chronic wounds are also characterized by senescent cells which have decreased proliferative and secretory capacities and decreased responsiveness to the wound healing signals. ${ }^{10-13}$

All chronic wounds naturally contain microorganisms. Microbial involvement of a wound can be described in the terms of the wound infection continuum: contamination, colonization, local infection, spreading infection, and systemic infection. ${ }^{14}$ Clinically, a chronic wound infection can present with impaired healing, unhealthy granulation tissue, putrid odor, increased exudate, erythema $>1-2 \mathrm{~cm}$, warmth around the wound, and necrotic tissue. ${ }^{15}$ The majority of chronic wounds have been reported to consist of a predominantly polymicrobial flora composed of aerobes and anaerobes, although, delayed wound healing with or without clinical signs of infection was more commonly associated with aerobic or facultative pathogens. Staphylococcus aureus, Pseudomonas aeruginosa, and beta-hemolytic Streptococci were reported as the most common pathogens causing chronic wound infections in some earlier studies. ${ }^{16}$

Evidence suggests that biofilms are present in over $90 \%$ of chronic wounds. ${ }^{17}$ During the inflammatory response to wound infection, leukocytes attach to the biofilm and release enzymes that propagate the inflammatory response and affects the healing processes, leading to the persistence of chronic wound. ${ }^{18-23}$ Biofilms exhibit the ability to mutate and alter their sensitivity to antibacterial agents. Planktonic bacteria are released from the biofilm onto the wound bed forming new colonies that can lead to local infection or weakening of the collagen matrix. ${ }^{24,25}$

Over the years, increasing resistance to commonly used antibiotics has been seen in chronic wounds. ${ }^{3}$ The increasing prevalence of multidrug-resistant organisms has complicated the choice of selecting an appropriate antibiotic for treating chronic wound infections. An understanding of the challenges in the management of chronic wounds and effectively addressing them will lead to a better outcome in terms of improved quality of life of the patients, reduced morbidity, mortality, and decreased healthcare costs. Figure 1 shows the various types of chronic wounds that are usually treated at our unit.

The objective of this study was to analyze the demographic, clinical, and microbiological profiles of patients presenting to our unit with chronic wounds of various etiologies. We intend to present a current overview of chronic wounds with regards to causes, clinical features, organisms isolated, antibiotic sensitivity profiles, treatments administered, and their outcomes.

\section{Patients and Methods Study Design}

We performed a prospective observational study of patients presenting to us with chronic wounds over a period of one year, from October 2018 to September 2019. The study was conducted at the Department of Burns and Plastic Surgery of a tertiary care institute in a non-metropolitan city in Central India.

\section{Inclusion Criteria}

Patients who presented to us with chronic wounds ( $\geq 3$ weeks duration) of any etiology and were willing to participate in the study.

\section{Exclusion Criteria}

None.

\section{Method}

Permission for conducting the study was obtained from the Institutional Human Ethics Committee (no. IHEC-LOP/ 2018/IM0194). The study protocol conformed to the ethical guidelines of the 1975 Declaration of Helsinki. Written informed consent was obtained from all the participants before their inclusion in the study. Data collected from the patients included demographic details, history, clinical features, and relevant laboratory reports. 

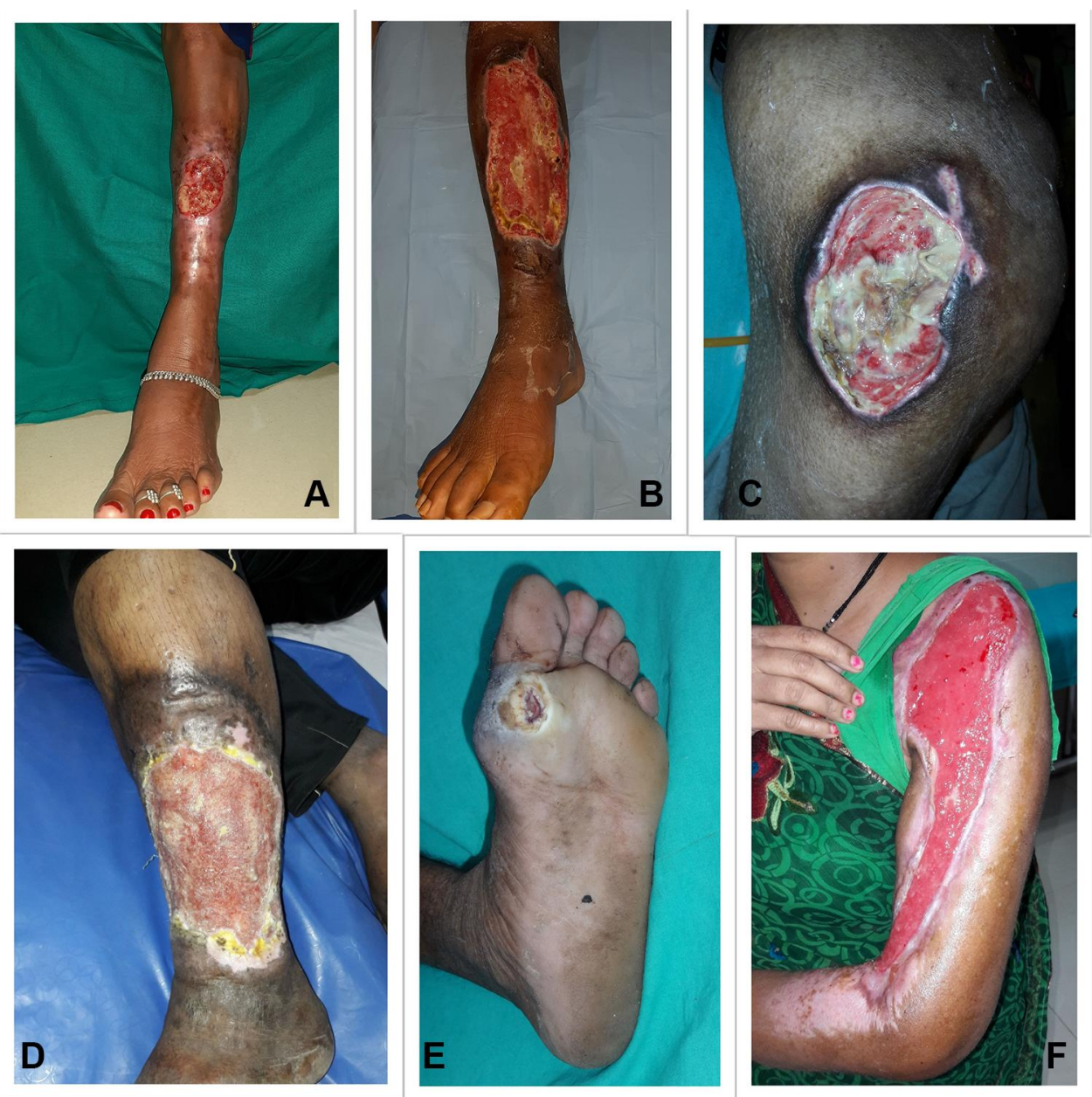

Figure I Spectrum of chronic wounds presenting to our unit. (A) Long-standing post-traumatic chronic wound over leg region. (B) Chronic wound on the leg following cellulitis. (C) Trochanteric pressure sore. (D) Venous ulcer in the gaiter area. (E) Diabetic foot ulcer. (F) Chronic wound following a thermal burn.

After assessment and photographic documentation, a sample was taken from the wound for culture and sensitivity studies. When a patient presented with multiple wounds, the largest wound was selected for sampling and analysis. An area near the center of the wound free of necrotic tissue and debris was pre-cleaned with non-bacteriostatic saline. Then, the end of a culture swab was rotated over a $1 \mathrm{~cm}^{2}$ area for 5 seconds with sufficient pressure to extract fluid from within the wound tissue (Levine's technique). ${ }^{26}$ All specimens were appropriately labeled and dispatched together with patient information sheets for aerobic culture and antibiotic sensitivity studies. All the specimens were analyzed by the same microbiological laboratory. Standard antibiotic susceptibility testing was performed for the most commonly used antibiotics.

Empirical treatment was instituted if needed, according to the clinical picture and severity at presentation. Once culture and sensitivity reports were available, treatment was instituted or modified, as necessary. Local wound treatment was performed with sharp/mechanical debridements followed by dressings with different agents. The progress was monitored until the wound healed, either by conservative management or by surgical intervention. STSG (Split-thickness skin grafts), local and distant flaps were the various surgical interventions performed. Patients were followed-up for six months after the wound healed.

\section{Statistical Analysis}

All the patient and wound data were entered in a spreadsheet created in Microsoft Excel ${ }^{\mathrm{TM}}$ software 2013 version (CMicrosoft Corporation, Redmond, Washington, United States). Data analysis was carried out using Epi Info software version 7.2 (Epi Info $^{\mathrm{TM}}$, Centers for Disease Control and Prevention, Atlanta, Georgia). Nominal variables were statistically described with frequencies and percentages. Continuous variables were described with means and standard deviations. Ordinal variables were also presented in the form of frequencies and percentages. 


\section{Results}

A total of 103 patients were included in the study (Table 1). There were 79 (76.69\%) males and 24 (23.3\%) females, with a male to female ratio of 3.29:1. The age of the patients ranged from 3 to 86 years with a mean age and standard deviation of 40.96 years and 16.307 , respectively. Trauma and infection were the most frequent causes of chronic wounds in the study. Majority of the patients presented with lower limb wounds $(\mathrm{n}=81,78.64 \%)$. The size of the index wound ranged from $1.57 \mathrm{~cm}^{2}$ to $675 \mathrm{~cm}^{2}$ at presentation.

Swab specimens from 103 wounds were cultured and 99 pathogenic organisms belonging to 17 different species were isolated (Table 2). Seventy-five wounds (72.81\%) had growth of a single organism from the wound. Among the isolates, gram-negative organisms were more common compared to gram-positive organisms (62 and 37 isolates, respectively). Staphylococcus aureus was the most common species isolated with 30 isolates (30 of 99, $30.30 \%)$. There were $20 \quad(20.20 \%)$ isolates of Pseudomonas aeruginosa.

Antibiotic sensitivity profiles of the isolates to commonly used antibiotics were analyzed. Among the MSSA (methicillin-sensitive Staphylococcus aureus) strains, 77.77\% (14 of 18 ) isolates were either sensitive or had intermediate sensitivity to Co-trimoxazole; $88.88 \%$ isolates were either sensitive or had intermediate sensitivity to Tetracycline and Clindamycin. Around $61.11 \%$ MSSA isolates showed resistance to Levofloxacin, $77.77 \%$ isolates showed resistance to Ciprofloxacin, and $55.55 \%$ isolates showed resistance to Erythromycin.

In MRSA (methicillin-resistant Staphylococcus aureus) isolates, $91.66 \%$ (11 of 12) isolates were sensitive to Clindamycin, $66.66 \%$ isolates were either sensitive or had intermediate sensitivity to Erythromycin, and all isolates were sensitive to Vancomycin. Around $91.66 \%$ MRSA isolates were resistant to Penicillin, Ciprofloxacin, and Levofloxacin; $58.33 \%$ isolates were resistant to Cotrimoxazole.

Among Pseudomonas aeruginosa strains isolated, 95\% (19 of 20) were either sensitive or had intermediate sensitivity to Piperacillin+Tazobactam, $85 \%$ isolates were either sensitive or had intermediate sensitivity to Gentamycin, $80 \%$ isolates were sensitive to Ciprofloxacin, and $60 \%$ isolates were sensitive to Cefepime. Thirty percent isolates of Pseudomonas
Table I Demographic and Clinical Characteristics of the Patients

\begin{tabular}{|c|c|}
\hline Characteristics & No. of patients n (\%) \\
\hline \multicolumn{2}{|l|}{ I. Sex } \\
\hline Males & 79 (76.69) \\
\hline Females & $24(23.3)$ \\
\hline Male:Female ratio & $3.29: 1$ \\
\hline 2. Age in years: mean (SD) & $\begin{array}{l}40.96 \text { ( } 16.307) \text {; age range: } 3- \\
86 \text { years }\end{array}$ \\
\hline \multicolumn{2}{|l|}{$\begin{array}{l}\text { 3. Duration of wound in weeks } \\
\text { category }\end{array}$} \\
\hline$\leq 12$ weeks & $60(58.25)$ \\
\hline$>12$ to 24 weeks & 14 (I3.59) \\
\hline$>24$ to 52 weeks & $14(13.59)$ \\
\hline$>52$ to 104 weeks & $12(11.65)$ \\
\hline$>104$ to 156 weeks & $3(2.91)$ \\
\hline \multicolumn{2}{|l|}{ 4. Etiology } \\
\hline Trauma & $29(28.15)$ \\
\hline Infection & $25(24.27)$ \\
\hline Burns & 14 (I3.59) \\
\hline a) Scald & 7 (6.79) \\
\hline b) Thermal & $3(2.91)$ \\
\hline c) Electrical & $3(2.91)$ \\
\hline d) Chemical & I (0.97) \\
\hline Venous incompetence & II (I0.67) \\
\hline Diabetic foot & $9(8.73)$ \\
\hline Pressure sore & $7(6.79)$ \\
\hline Unstable scar & $5(4.85)$ \\
\hline Hansen's & $2(1.94)$ \\
\hline Marjolin's ulcer & I $(0.97)$ \\
\hline \multicolumn{2}{|l|}{$\begin{array}{l}\text { 5. Number of wounds at } \\
\text { presentation }\end{array}$} \\
\hline One & $89(86.4)$ \\
\hline Two & II (I0.67) \\
\hline Three or more & $3(2.91)$ \\
\hline \multicolumn{2}{|l|}{ 6. Site } \\
\hline Head and Neck & $5(4.85)$ \\
\hline Upper Limb & $9(8.73)$ \\
\hline Trunk & $8(7.76)$ \\
\hline Lower Limb & 81 (78.64) \\
\hline \multicolumn{2}{|l|}{ 7. Wound size in $\mathrm{cm}^{2}$ categories } \\
\hline $1-10$ & $30(29.12)$ \\
\hline $10.1-20$ & $13(12.62)$ \\
\hline $20.1-50$ & $17(16.5)$ \\
\hline $50.1-100$ & $21(20.38)$ \\
\hline $100.1-200$ & $17(16.5)$ \\
\hline $200.1-500$ & $3(2.91)$ \\
\hline$>500$ & $2(1.94)$ \\
\hline
\end{tabular}

Abbreviation: SD, standard deviation. 
Table 2 Organisms Isolated

\begin{tabular}{|l|l|}
\hline Characteristics & Result \\
\hline I. No. of isolates per wound & No. of patients n (\%) \\
One & $75(72.81)$ \\
Two & $11(10.67)$ \\
Three or more & $8(7.76)$ \\
No Growth & $9(8.73)$ \\
\hline 2. Gram staining of the isolates & No. of isolates n (\%) \\
Total no. of isolates & 99 \\
Gram-positive organisms & $37(37.37)$ \\
Gram-negative organisms & $62(62.62)$ \\
\hline 3. Organism & No. of isolates n \\
Staphylococcus aureus & 30 \\
a) MSSA & 18 \\
b) MRSA & 12 \\
Pseudomonas aeruginosa & 20 \\
Klebsiella pneumoniae & 12 \\
Escherichia coli & 10 \\
Proteus mirabilis & 8 \\
Enterobacter species & 4 \\
Acinetobacter baumanii & Streptococcus pyogenes \\
Enterococcus species & 2 \\
Proteus vulgaris, Proteus hauseri & 2 \\
\hline $\begin{array}{l}\text { Providencia stuartii, Citrobacter species, } \\
\text { Methicillin-resistant coagulase negative } \\
\text { Staphylococcus, Klebsiella oxytoca, } \\
\text { Methicillin-resistant Staphylococcus } \\
\text { haemolyticus, Staphylococcus lugdunensis }\end{array}$ \\
\hline
\end{tabular}

Abbreviations: MSSA, Methicillin-sensitive Staphylococcus aureus; MRSA, Methicillinresistant Staphylococcus aureus.

aeruginosa were resistant to Ticarcillin+Clavulanate, Tobramycin, and Meropenem.

Table 3 presents the treatments used in the study subjects and complications. Most of the patients $(n=74,71.84 \%)$ needed surgical intervention for achieving wound closure. Among these, split-thickness skin grafting (STSG) was the most common surgical intervention performed, accounting for $43.68 \%(n=45)$ patients. Other procedures that were performed include random pattern local flaps, perforatorbased local flaps, muscle flaps, free flaps, and a cross-leg flap. Figures 2 and 3 show the pre-operative and post-operative pictures of the cases in which a surgical intervention (skin graft or flap) was performed. Twenty-nine (28.15\%) patients were treated conservatively.

Wound closure was achieved in most of the patients, except for four. One patient who presented with a longstanding previously undiagnosed Marjolin's ulcer of the
Table 3 Treatments and Complications

\begin{tabular}{|c|c|}
\hline Treatment Modality & $\begin{array}{l}\text { No. of Patients } n \\
\text { (\%) }\end{array}$ \\
\hline I. Conservative Management & $29(28.15)$ \\
\hline 2. Surgical intervention & $74(7 I .84)$ \\
\hline a) STSG & $45(43.68)$ \\
\hline b) Random pattern local flaps & $5(4.85)$ \\
\hline Transposition flap & I \\
\hline Rotation flap & I \\
\hline Limberg flap & I \\
\hline V-Y advancement flap & I \\
\hline Bilateral $\mathrm{V}$-Y advancement flap & I \\
\hline c) Perforator based local flaps & $18(17.47)$ \\
\hline Sural artery flap & 13 \\
\hline $\begin{array}{l}\text { Posterior tibial artery perforator based } \\
\text { fascio-cutaneous peninsular flap }\end{array}$ & 2 \\
\hline Pedicled ALT flap & I \\
\hline $\begin{array}{l}\text { Superior gluteal artery perforator based } \\
\text { pedicled islanded flap }\end{array}$ & I \\
\hline FDMA flap & I \\
\hline d) Muscle flaps & $2(1.94)$ \\
\hline Hemi-soleus flap & 1 \\
\hline Tensor fascia lata rotation advancement flap & I \\
\hline e) Free flaps & $2(1.94)$ \\
\hline Free ALT flap & 2 \\
\hline f) Cross-leg flap & I $(0.97)$ \\
\hline g) Above knee amputation & I $(0.97)$ \\
\hline \multicolumn{2}{|l|}{ Complications } \\
\hline Early & No. of patients $n$ \\
\hline $\begin{array}{l}\text { I. Small areas of graft loss treated } \\
\text { conservatively }\end{array}$ & 11 \\
\hline 2. Partial graft loss requiring re-grafting & 6 \\
\hline 3. Venous congestion in Sural artery flap & 2 \\
\hline $\begin{array}{l}\text { 4. Superficial necrosis in distal part of FDMA } \\
\text { flap }\end{array}$ & I \\
\hline 5. Total flap loss in free ALT flap & I \\
\hline Long term & \\
\hline Wounding in the skin grafts & 9 \\
\hline
\end{tabular}

Abbreviations: STSG, split-thickness skin grafting; ALT flap, anterolateral thigh flap; FDMA flap, first dorsal metatarsal artery flap.

leg region underwent an above-knee amputation. Three other patients who were kept on conservative management on an outpatient basis and had initial improvement in their wounds were lost to follow up.

\section{Discussion}

Trauma and infection accounted for causing the majority $(52.42 \%)$ of the chronic wounds in our study population. This finding can be correlated with the male preponderance (76.69\%), as males are more susceptible to occupational and 

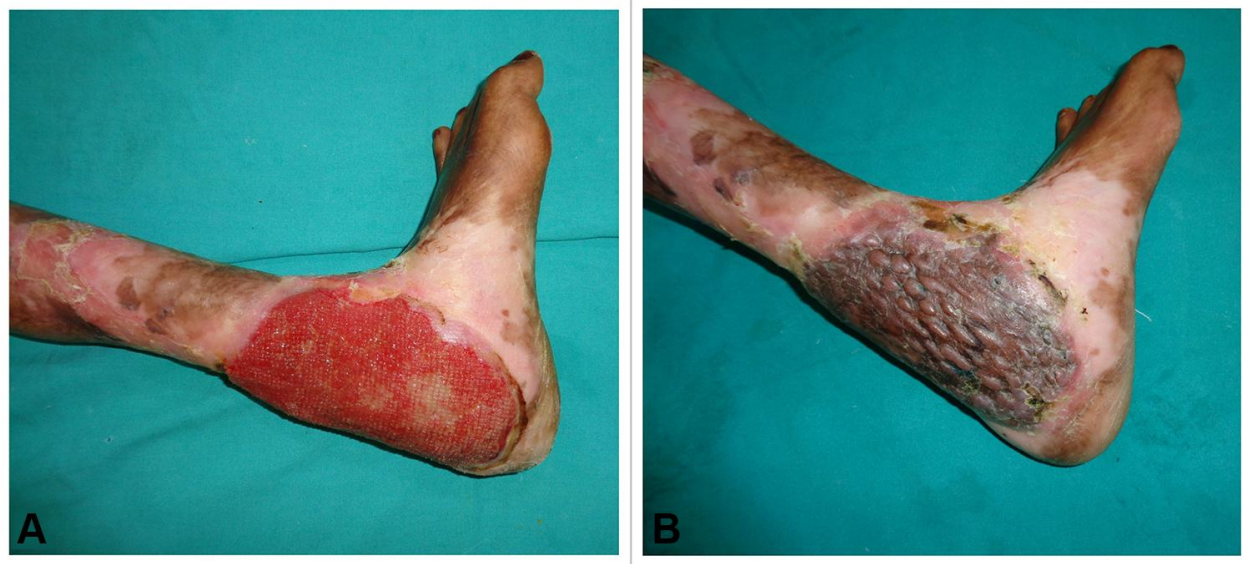

Figure 2 (A) Patient presenting with a post-thermal burn chronic wound over the posterior aspect of the leg. (B) Four weeks following split-thickness skin grafting with a well-settled graft.
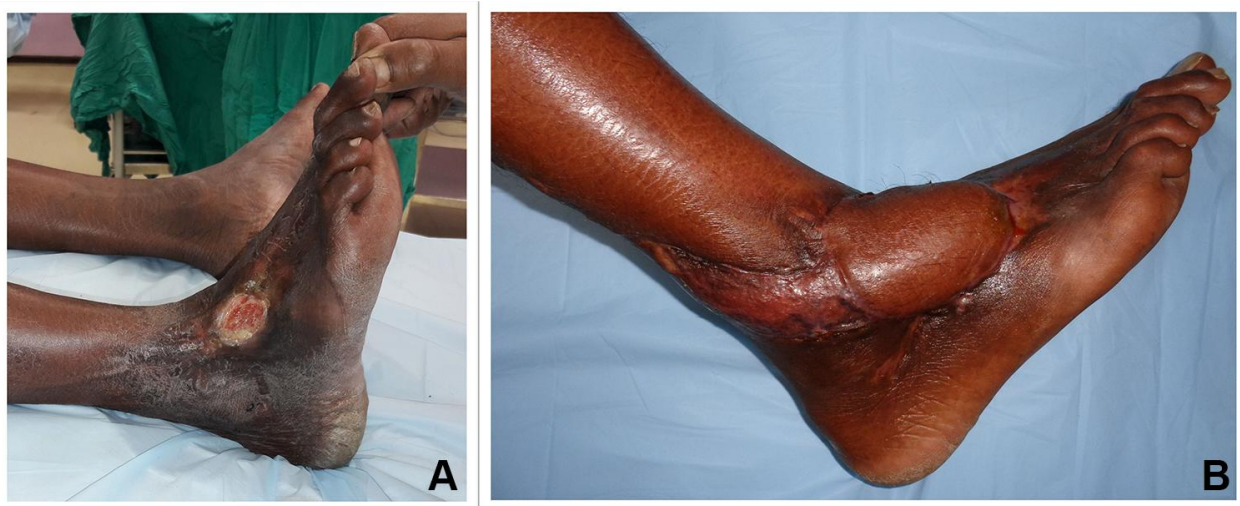

Figure 3 (A) Patient presenting with a long-standing post-traumatic chronic wound over the dorsum of the foot. (B) Two months following excision of the wound and reconstruction with islanded Sural artery flap.

accidental trauma. Burns, venous disease, diabetic foot, and pressure injuries were some of the other important causes. Gupta et al conducted a community-based cross-sectional study on the epidemiology of wounds and reported that untreated trauma, diabetes, and leprosy were the common causes of chronic wounds in their study population. ${ }^{27}$ This profile is different from that of western literature in which the majority of the chronic wounds are caused by venous disease followed by arterial disease.

In our study, most of the chronic wounds were located in the lower limbs (78.64\%). The vulnerability of the lower limbs for wounding has been consistently reported in many of the earlier studies. ${ }^{1,27-31}$ Most of the patients presented with a single wound. But 14 (13.58\%) patients had two or more wounds at presentation.

Identification of the causative organisms by microbial cultures and specific anti-microbial therapy are important measures in the management of chronic wound infections that are unresponsive to standard treatment. Many chronic wounds are indiscriminately treated with antibiotics because of the belief that high wound bioburden contributes to delayed healing. ${ }^{26}$ In the present study, we have used wound swabs obtained by Levine's technique for cultures. These swabs improve the ability to verify which of the chronic wounds really have high bioburden, thus decreasing the number of patients receiving unnecessary antibiotic treatment. The Levine method samples both the surface biofilm and from beneath the surface, ${ }^{24,26}$ and the culture results are comparable to those obtained by tissue biopsy. ${ }^{26,32,33}$ An international consensus update on wound infection published in 2016 also recommended Levine's technique as it was more effective than Z-swab technique for microbial cultures. ${ }^{14}$

Previous studies have shown that chronic wounds mostly have a polymicrobial flora. ${ }^{24}$ In our study, the 
majority $(n=75,72.81 \%)$ of the wounds had only one organism isolated from the wound in aerobic cultures. There were only $10.67 \%$ wounds with 2 species isolated from the wound and $7.76 \%$ wounds with 3 or more species isolated from the wound. No pathogenic organism was isolated in $8.73 \%$ wounds. Overall, there were more gram-negative organisms isolated than gram-positive organisms. We believe that these findings should be carefully considered when selecting empirical antibiotic therapy in patients presenting with severe infections. Empirical antibiotic therapy should also include coverage for MSSA or MRSA in patients at risk of these infections.

Angel et al reported that Staphylococcus aureus and Pseudomonas aeruginosa are the most commonly isolated pathogens in chronic wounds. ${ }^{24}$ Our study revealed similar findings. Staphylococcal species comprised $33.3 \%$ of all isolates recovered; $90.9 \%$ of these isolates comprised Staphylococcus aureus. Pseudomonas aeruginosa was the second most common species isolated comprising $20.20 \%$ isolates.

The emergence of community-acquired MRSA has been previously studied. ${ }^{34,35}$ In our study, cultures of specimens from $12(11.65 \%)$ patients had grown MRSA. We found that $91.66 \%$ MRSA isolates were susceptible to Clindamycin and $100 \%$ isolates were susceptible to Vancomycin. Vancomycin should be considered as the drug of choice for treating MRSA infections caused by multi-drug resistant strains. Clindamycin can be considered as an alternative in less severe cases. We have also found that $91.66 \%$ of MRSA strains were resistant to Ciprofloxacin and Levofloxacin. Some earlier studies also reported $80 \%$ quinolone resistance in MRSA. ${ }^{36}$ Hence, quinolones should not be considered as an option for empirical therapy, in cases where MRSA is suspected. Even in cases of MSSA, we do not recommend therapy with Quinolones considering the degree of resistance among the isolates $(77.77 \%$ isolates resistant to Ciprofloxacin and $61.11 \%$ resistant to Levofloxacin).

Valencia et al reported in their study that more than half (56\%) of the Pseudomonas aeruginosa isolates from leg ulcers were resistant to quinolones. ${ }^{30}$ In contrast, we found out that in our study subjects, Pseudomonas aeruginosa isolates were largely sensitive to quinolones $(80 \%$ isolates were sensitive to Ciprofloxacin and 75\% isolates were sensitive to Levofloxacin). Therefore, we propose that quinolones can be considered as an option for the empirical treatment of suspected Pseudomonas aeruginosa infections of chronic wounds. We have also found that the frequency of infections caused by other gram-negative organisms such as Klebsiella pneumoniae, Escherichia coli, and Proteus mirabilis are increasing.

In our study, apart from the anti-microbial therapy to treat wound infections, wound bed preparation was done with a combination of sharp/mechanical debridements followed by dressings with different agents (Table S1, supple mentary data). Nanocrystalline silver gel was the most common dressing agent used in the study patients. Madhusudhan performed a prospective randomized controlled trial in pseudomonal wound infections and concluded that $1 \%$ acetic acid is a simple, safe and effective topical antiseptic that can be used in the elimination of $P$. aeruginosa from chronic-infected wounds. ${ }^{37}$

Sharp/mechanical debridements were performed in almost all of our study patients. Attempts were made to perform debridement at every dressing until the wound was grossly free from slough. Necrotic tissue in the form of slough or eschar is very commonly found in chronic wounds. $^{38}$ It not only affects wound healing but also heightens the risk of infection. ${ }^{39}$ Therefore, debridements are required to get rid of the non-viable tissue and disrupt the biofilms. Appropriate debridement helps accelerate the wound healing process, by removing the barrier of nonviable tissue. ${ }^{40}$ It also helps to break down the defenses of the microbes, exposing them, and permitting more effective action of topical antimicrobials. ${ }^{41}$ The importance of surgical debridement in wound bed preparation and its role in disrupting biofilms has been highlighted in the recent international consensus studies. ${ }^{42,43}$ In our study, $28.15 \%$ of patients underwent conservative management alone, which consisted of debridements, dressings, and antimicrobials, when needed.

Around $71.84 \%$ patients underwent surgical intervention, in addition to conservative management. The coverage provided included split-thickness skin grafts, local and distant flaps. Langer et al performed a prospective study on the adjunctive role of NPWT (Negative-pressure wound therapy) in the healing of chronic wounds. ${ }^{44} \mathrm{~A}$ significant observation from this study is that, in their study group of 60 patients, they identified that coverage in the form of a flap was required at presentation in $63.33 \%$ of patients with chronic wounds. Hence, we can infer that where available, early referral to reconstructive services may shorten the course and improve the outcome in chronic wounds. Further, in this study, after the institution of NPWT, $60 \%$ of the patients had wound healing by secondary intention, and $40 \%$ of patients required split- 
thickness skin grafts. ${ }^{44}$ In our study, STSG was performed in $43.68 \%$ of the patients.

Our study had some limitations. We did not employ methods to isolate anaerobic organisms and fungi because of some logistical constraints. Also, the small sample size in our study may not be enough to draw general conclusions applicable to all types of chronic wounds in different settings. Further studies with larger sample sizes are necessary.

\section{Conclusion}

Chronic wounds impose an enormous burden on the patients and healthcare systems around the world. Knowledge regarding the causative factors, prevalent organisms in chronic wound infections, antibiotic susceptibility patterns of the isolates, and treatment options is extremely useful to all the people involved in patient care, infection control, healthcare administration, and planning. Our study gives a current overview of the causes, clinical presentation, prevalent microbial flora, and their antibiotic susceptibilities in the chronic wounds presenting to our unit. Treatments administered are discussed with emphasis on the different reconstructions performed.

\section{Abbreviations}

ALT flap, anterolateral thigh flap; FDMA flap, first dorsal metatarsal artery flap; MMPs, matrix metalloproteinases; MRSA, methicillin-resistant Staphylococcus aureus; MSSA, methicillin-sensitive Staphylococcus aureus; NPWT, negative pressure wound therapy; SD, standard deviation; STSG, split-thickness skin grafting.

\section{Acknowledgments}

We acknowledge the contributions of the staff and residents of our department in conducting this study.

\section{Author Information}

Dr. Manal M Khan, MBBS, MS, MCh, is a Senior Consultant Plastic Surgeon, Additional Professor, and Head of the Department of Burns and Plastic Surgery, All India Institute of Medical Sciences (AIIMS), Bhopal. Dr. Ved Prakash Rao Cheruvu, MBBS, MS, MCh, FCAPS, is a Consultant Plastic Surgeon and Assistant Professor of Plastic Surgery at the Department of Burns and Plastic Surgery, AIIMS Bhopal. Dr Deepak Krishna, MBBS, MS, MCh, is a Consultant Plastic Surgeon and Associate Professor of Plastic Surgery at the Department of Burns and Plastic Surgery, AIIMS Bhopal. Dr Reena Minz, MBBS, MS, is a Senior Resident at the Department of Burns and Plastic Surgery, AIIMS Bhopal. Dr Michael Laitonjam, MBBS, MS, is a Senior Resident at the Department of Burns and Plastic Surgery, AIIMS Bhopal. Dr Rishabh Joshi, MBBS, MS, is a Senior Resident at the Department of Burns and Plastic Surgery, AIIMS, Bhopal.

\section{Funding}

We have not received any financial or material support for conducting this study.

\section{Disclosure}

The authors report no conflicts of interest, financial or otherwise, in relation to this work.

\section{References}

1. Frank DN, Wysocki A, Specht-Glick DD, et al. Microbial diversity in chronic open wounds. Wound Repair Regen. 2009;17(2):163-172. doi:10.1111/j.1524-475X.2009.00472.x

2. Guest JF, Ayoub N, McIlwraith T, et al. Health economic burden that wounds impose on the national health service in the UK. BMJ Open. 2015;5(12):e009283. doi:10.1136/bmjopen-2015-009283

3. Brownrigg JRW, Apelqvist J, Bakker K, Schaper NC, Hinchliffe RJ. Evidence-based management of PAD \& the diabetic foot. Eur J Vasc Endovasc Surg. 2013;45(6):673-681. doi:10.1016/j.ejvs.2013.02.014

4. Richmond NA, Maderal AD, Vivas AC. Evidence-based management of common chronic lower extremity ulcers. Dermatol Ther. 2013;26 (3):187-196. doi:10.1111/dth.12051

5. Reiber G, Lipsky B, Gibbons G. The burden of diabetic foot ulcers. Am J Surg. 1998;176(2):5S-10S. doi:10.1016/S0002-9610 (98)00181-0

6. Lawrence CP. The infected decubitus ulcer: superman's kryptonite. Ski Dermatol Clin. 2005;4(1):7-8.

7. Guo S, DiPietro LA. Factors affecting wound healing. J Dent Res. 2010;89(3):219-229. doi:10.1177/0022034509359125

8. Landén NX, Li D, Ståhle M. Transition from inflammation to proliferation: a critical step during wound healing. Cell Mol Life Sci. 2016;73(20):3861-3885.

9. Parnham A, Bousfield C. The influence of matrix metalloproteases and biofilm on chronic wound healing: a discussion. Br J Community Nurs. 2018;23(Suppl Sup3):S22-29. doi:10.12968/bjen.2018.23. Sup3.S22

10. Schultz GS, Sibbald RG, Falanga V, et al. Wound bed preparation: a systematic approach to wound management. Wound Repair Regen. 2003;11(Suppl 1):S1-S28. doi:10.1046/j.1524-475X.11.s2.1.x

11. Bourguignon LYW. Matrix hyaluronan-activated CD44 signaling promotes keratinocyte activities and improves abnormal epidermal functions. Am J Pathol. 2014;184(7):1912-1919. doi:10.1016/j. ajpath.2014.03.010

12. Cook H, Stephens P, Davies KJ, Thomas DW, Harding KG. Defective extracellular matrix reorganization by chronic wound fibroblasts is associated with alterations in TIMP-1, TIMP-2, and MMP-2 activity. $J$ Invest Dermatol. 2000;115(2):225-233. doi:10.1046/j.15231747.2000.00044.x

13. Wall IB, Moseley R, Baird DM, et al. Fibroblast dysfunction is a key factor in the non-healing of chronic venous leg ulcers. $J$ Invest Dermatol. 2008;128(10):2526-2540. doi:10.1038/jid.2008.114 
14. International Wound Infection Institute (IWII). Wound infection in clinical practice. Wound Int. 2016.

15. Gardner SE, Frantz RA, Doebbeling BN. The validity of the clinical signs and symptoms used to identify localized chronic wound infection. Wound Repair Regen. 2001;9(3):178-186. doi:10.1046/j.1524475x.2001.00178.x

16. Bowler PG, Davies BJ. The microbiology of infected and noninfected leg ulcers. Int J Dermatol. 1999;38(8):573-578. doi:10.1046/j.13654362.1999.00738.x

17. Attinger $\mathrm{C}$, Wolcott R. Clinically addressing biofilm in chronic wounds. Adv Wound Care. 2012;1(3):127-132. doi:10.1089/wound.2011.0333

18. Camilli A, Bassler BL. Bacterial small-molecule signaling pathways. Science. 2006;311(5764):1113-1116. doi:10.1126/science.1121357

19. Nadell CD, Xavier JB, Levin SA, Foster KR. The evolution of quorum sensing in bacterial biofilms. PLoS Biol. 2008;6(1):e14. doi:10.1371/journal.pbio.0060014

20. Jensen PØ, Bjarnsholt T, Phipps R, et al. Rapid necrotic killing of polymorphonuclear leukocytes is caused by quorum-sensing-controlled production of rhamnolipid by pseudomonas aeruginosa. Microbiology. 2007;153(5):1329-1338. doi:10.1099/mic.0.2006/003863-0

21. Walker TS, Tomlin KL, Worthen GS, et al. Enhanced pseudomonas aeruginosa biofilm development mediated by human neutrophils. Infect Immun. 2005;73(6):3693-3701. doi:10.1128/IAI.73.6.3693-3701.2005

22. Bjarnsholt T, Alhede M, Alhede M, et al. The in vivo biofilm. Trends Microbiol. 2013;21(9):466-474. doi:10.1016/j.tim.2013.06.002

23. Copeland-Halperin LR, Kaminsky AJ, Bluefeld N, Miraliakbari R. Sample procurement for cultures of infected wounds: a systematic review. J Wound Care. 2016;25(Suppl 4):S4-S10. doi:10.12968/ jowc.2016.25.Sup4.S4

24. Angel DE, Lloyd P, Carville K, Santamaria N. The clinical efficacy of two semi-quantitative wound-swabbing techniques in identifying the causative organism(s) in infected cutaneous wounds. Int Wound J. 2011;8(2):176-185. doi:10.1111/j.1742-481X.2010.00765.x

25. Potera C. Forging a link between biofilms and disease. Science. 1999;283(5409):1837-1839. doi:10.1126/science.283.5409.1837

26. Gardner SE, Frantz RA, Saltzman CL, Hillis SL, Park H, Scherubel M. Diagnostic validity of three swab techniques for identifying chronic wound infection. Wound Repair Regen. 2006;14(5):548557. doi:10.1111/j.1743-6109.2006.00162.x

27. Gupta N, Gupta SK, Shukla VK, Singh SP. An Indian communitybased epidemiological study of wounds. J Wound Care. 2004;13 (8):323-325. doi:10.12968/jowc.2004.13.8.26657

28. Howell-Jones RS, Wilson MJ, Hill KE, Howard AJ, Price PE, Thomas DW. A review of the microbiology, antibiotic usage and resistance in chronic skin wounds. $J$ Antimicrob Chemother. 2005;55(2):143-149. doi:10.1093/jac/dkh513

29. Mekkes JR, Loots MAM, Van Der Wal AC, Bos JD. Causes, investigation and treatment of leg ulceration. Br J Dermatol. 2003;148 (3):388-401. doi:10.1046/j.1365-2133.2003.05222.x

30. Quatresooz P, Henry F, Paquet P, Pierard-Franchimont C, Harding K, Pierard GE. Deciphering the impaired cytokine cascades in chronic leg ulcers (review). Int J Mol Med. 2003;11(4):411-418.
31. Valencia IC, Kirsner RS, Kerdel FA. Microbiologic evaluation of skin wounds: alarming trend toward antibiotic resistance in an inpatient dermatology service during a 10-year period. J Am Acad Dermatol. 2004;50(6):845-849. doi:10.1016/j.jaad.2003.11.064

32. Slater RA, Lazarovitch T, Boldur I, et al. Swab cultures accurately identify bacterial pathogens in diabetic foot wounds not involving bone. Diabet Med. 2004;21(7):705-709. doi:10.1111/j.14645491.2004.01221.x

33. Davies CE, Hill KE, Wilson MJ, et al. Use of $16 \mathrm{~S}$ ribosomal DNA PCR and denaturing gradient gel electrophoresis for analysis of the microfloras of healing and nonhealing chronic venous leg ulcers. $J$ Clin Microbiol. 2004;42(8):3549-3557. doi:10.1128/JCM.42.8.35493557.2004

34. Diekema DJ, Pfaller MA, Schmitz FJ, et al. Survey of infections due to staphylococcus species: frequency of occurrence and antimicrobial susceptibility of isolates collected in the United States, Canada, Latin America, Europe, and the western pacific region for the sentry antimicrobial surveillance program, 1997-1999. Clin Infect Dis. 2001;32 (Suppl 2):S114-S132. doi:10.1086/320184

35. Moran GJ, Krishnadasan A, Gorwitz RJ, et al. Methicillin-resistant S. aureus infections among patients in the emergency department. $N$ Engl J Med. 2006;355(7):666-674. doi:10.1056/NEJMoa055356

36. Neu HC. The crisis in antibiotic resistance. Science. 1992;257 (5073):1064-1073. doi:10.1126/science.257.5073.1064

37. Madhusudhan VL. Efficacy of $1 \%$ acetic acid in the treatment of chronic wounds infected with pseudomonas aeruginosa: prospective randomised controlled clinical trial. Int Wound J. 2016;13(6):11291136. doi:10.1111/iwj.12428

38. Nunan R, Harding KG, Martin P. Clinical challenges of chronic wounds: searching for an optimal animal model to recapitulate their complexity. Dis Model Mech. 2014;7(11):1205-1213. doi:10.1242/dmm.016782

39. Snyder R, Fife C, Moore Z. Components and quality measures of DIME (devitalized tissue, infection/inflammation, moisture balance, and edge preparation) in wound care. Adv Skin Wound Care. 2016;29 (5):205-215. doi:10.1097/01.ASW.0000482354.01988.b4

40. Atkin L. Chronic wounds: the challenges of appropriate management. Br J Community Nurs. 2019;24(Suppl 9):S26-S32. doi:10.12968/ bjcn.2019.24.Sup9.S26

41. Bowler PG, Parsons D. Combatting wound biofilm and recalcitrance with a novel anti-biofilm hydrofiber $\left.{ }^{(}\right)$wound dressing. Wound Med. 2016;14:6-11. doi:10.1016/j.wndm.2016.05.005

42. Schultz G, Bjarnsholt T, James GA, et al. Consensus guidelines for the identification and treatment of biofilms in chronic nonhealing wounds. Wound Repair Regen. 2017;25(5):744-757. doi:10.1111/wrr.12590

43. Murphy C, Atkin L, Swanson T, et al. International consensus document. Defying hard-to-heal wounds with an early antibiofilm intervention strategy: wound hygiene. J Wound Care. 2020;29(Suppl 3b): S1-S28. doi:10.12968/jowc.2020.29.Sup3b.S1

44. Langer V, Bhandari PS, Rajagopalan S, Mukherjee MK. Negative pressure wound therapy as an adjunct in healing of chronic wounds. Int Wound J. 2015;12(4):436-442. doi:10.1111/iwj.12132
Chronic Wound Care Management and Research

\section{Publish your work in this journal}

Chronic Wound Care Management and Research is an international, peer reviewed, open access, online journal publishing original research, reviews, editorials, and commentaries on the causes and management of chronic wounds and the major issues related to chronic wound management. Topics also include chronic wounds as comorbidities to other conditions, patient adherence to therapy, and the economic burden of chronic wounds. The manuscript management system is completely online and includes a very quick and fair peer review system, which is all easy to use. Visit http://www.dovepress.com/ testimonials.php to read real quotes from published authors. 